Easy Hi-C: A simple efficient protocol for 3D genome mapping in small cell populations

Leina $\mathrm{Lu}^{1}$, Xiaoxiao $\mathrm{Liu}^{1}$, Jun Peng ${ }^{1}$, Yan Li ${ }^{1, \#}$, Fulai Jin ${ }^{1,2, \#}$

${ }^{1}$ Department of Genetics and Genome Sciences, Case Western Reserve University, 10900 Euclid Avenue, Cleveland, Ohio 44106, USA

${ }^{2}$ Case Comprehensive Cancer Center, Case Western Reserve University, 10900 Euclid Avenue, Cleveland, Ohio 44106, USA

\# Correspondence: fxj45@case.edu (F.J.) or yxl1379@case.edu (Y.L.)

Despite the growing interest in studying the mammalian genome organization, it is still challenging to map the DNA contacts genome-wide. Here we present easy Hi-C (eHi-C), a highly efficient method for unbiased mapping of 3D genome architecture. The eHi-C protocol only involves a series of enzymatic reactions and maximizes the recovery of DNA products from proximity ligation. We show that eHi-C can be performed with 0.1 million cells and yields high quality libraries comparable to Hi-C.

The invention of $\mathrm{Hi}-\mathrm{C}$ has transformed our understanding of the mammalian genome organization $^{1,2}$. In the past few years, with increasing sequencing depth, a hierarchy of genome structures at different length scales has been revealed. With $\sim 10$ million $\mathrm{Hi}-\mathrm{C}$ reads at $1 \mathrm{Mb}$ resolution, it was first discovered that human genome is 
organized into two compartments (compartment $A / B$ ) reflecting the spatial separation of euchromatin and heterochromatin ${ }^{1}$. Later, with a few hundred million reads and at $\sim 50 \mathrm{~kb}$ resolution, it was discovered that the mammalian genome is partitioned into $\mathrm{Mb}$ sized topological associated domains (TADs), which are largely invariant between different cell types, and even conserved between mammalian species ${ }^{3-7}$. Most recently, kilobase resolution $\mathrm{Hi}-\mathrm{C}$ analysis was achieved with sequencing depth at billion level ${ }^{8,}$ 9. At this resolution, cell type-specific chromatin loops within TADs between cisregulatory elements ${ }^{8,10-13}$, including promoters, enhancers, silencers and insulators, can be discerned. Apparently, with continuous improvement of sequencing technology, high-depth $\mathrm{Hi}-\mathrm{C}$ data will become preferable because they can reveal genome organization with full detail.

However, generating high quality $\mathrm{Hi}-\mathrm{C}$ library for deep sequencing is often challenging, especially when the amount of starting material is small. In $\mathrm{Hi}-\mathrm{C}$ protocol, 5' overhangs are created after restrictive DNA digestion (e.g. with HindIII) so that ligation junctions can be labeled with biotinylated nucleotides and eventually enriched in a pull-down step with streptavidin beads. However, this biotin-dependent strategy has several intrinsic limitations that affect library quality: (a) The efficiency of biotin incorporation into DNA is only $\sim 20-30 \%$, sometimes as low as $5 \%^{14}$, therefore a majority of ligation junctions are not labeled; (b) Only a portion of labeled ligation junction products can be recovered after several washes, further decreasing the library complexity; (c) Biotin-pulldown may not completely remove contamination from unligated DNA products. Due to these reasons, Hi-C library from $\sim 10$ million cells usually 
start to reach saturation after yielding several hundred million reads, and multiple biological replicates are necessary for kilobase-resolution $\mathrm{Hi}-\mathrm{C}$ analysis. Therefore, most published $\mathrm{Hi}-\mathrm{C}$ analysis are performed in cells or tissues where sample abundance is not a limiting factor.

We reasoned that we may circumvent the limitations of $\mathrm{Hi}-\mathrm{C}$, thus improve the assay efficiency by using a biotin-free strategy to enrich ligation products. Inspired by the well-established $4 \mathrm{C}$ method $^{15}$, we developed easy Hi-C, which only involves a series of enzymatic reactions to generate DNA libraries for the study of genome architecture (Fig. 1a). In this new protocol, we begin with the in situ proximity ligation procedure and perform HindIII digestion and proximity ligation while keeping nuclei intact. It has been shown that comparing to "in-solution ligation" (ligation after nuclear lysis), the in situ ligation approach can reduce false positive interactions ${ }^{9,16,17}$. In eHiC, ligation was performed directly after HindIII digestion without end repair, leading to HindIII-digestible junction products. After nuclear lysis and reverse crosslink, the DNA are digested with more frequent 4-base cutter Dpn/l before self-ligation (Fig. 1a). The samples are next treated with exonuclease to remove DNA that failed to form selfcircles, and contaminations from un-ligated ends and other linear DNA species (Fig. 1a). In order to enrich the ligation junction products, we then employ a simple trick by cutting the self-circularized DNA again with HindIII, and only re-linearized junction DNA will be amplified during the library generation (Fig. 1a).

Because all the reads from eHi-C library are next to HindIIl sites (Fig. 1a), the reproducible ligation events between the same pair of HindIII ends will give rise to 
identical paired-end reads (Supplementary Fig. 1a). This can be problematic because read pairs from the reproducible ligation events cannot be distinguished from PCR duplications. To address this issue, we used a custom adapter with a random sequence as unique molecule index (UMI) (Fig. 1a, Supplementary Fig. 1b). Two identical paired-end reads are considered PCR duplicates only if they have the same UMI sequences (Supplementary Fig. 1b). It should be also noted that a similar approach named ELP was developed several years ago to identify DNA contacts in fission yeast $^{18}$. However, the design of ELP was flawed and cannot effectively remove contaminations from several species of non-junction DNA (Supplementary Fig. 2). Therefore, less than $4 \%$ of ELP reads represent proximity ligation events ${ }^{18}$. As mentioned above, by combining the in situ ligation procedure, an exonuclease treatment step, and the usage of UMI adapter (Fig. 1a), the eHi-C approach has solved the quality issues of ELP, and is suitable for the analysis of mammalian 3D genome.

We next tested the application of eHi-C in low-input setting with human primary lung fibroblast IMR90 cells. The eHi-C method uses more efficient "sticky end" ligation, and the protocol should havve a high recovery rate of ligation junction products because there is no DNA loss during the whole procedure (Fig. 1a). The only exception is the exonuclease digestion step: Ligation junction products may be digested if they fail to self-ligate (Fig. 1a). From a control experiment, we have determined that the efficiency of the self-ligation reaction is high ( $60 \%$, Supplementary Fig. 3), thus a majority of ligation products can be recovered for sequencing. We have generated multiple eHi-C libraries each with 0.1 0.2 million cells, and used low-depth or high- 
depth sequencing to evaluate the library quality (Supplementary Table 1). As expected, over $95 \%$ of mapped eHi-C reads begin with HindIII restrictive sequence AGCTT, indicating that nearly all reads are from re-linearized HindIII-digestible DNA circles. When one eHi-C library from 0.1 million cells is deep-sequenced to 150 million mapped read pairs, the percentage of PCR duplicates is low than all the 13 published IMR90 Hi-C libraries prepared with 100 times more (10 million) cells ${ }^{8}$ (Supplementary Table 1-2), indicating a significantly improved library complexity over Hi-C.

We next compared the sources of errors in $\mathrm{Hi}-\mathrm{C}$ and eHi-C libraries by examining the fractions of different types of paired-end reads ${ }^{8,14}$ (see Method, Supplementary Fig. 4). In Hi-C, read pairs fall in the same HindlII fragments are considered invalid, and the major type of invalid reads are "dangling reads" originated from non-ligation DNA (Supplementary Fig. 4, Supplementary Table 2). In contrast, the only type of invalid pairs from eHi-C are self-circles (Supplementary Fig. 4b), all the other types of invalid pairs are removed by exonuclease treatment. One drawback of eHi-C is that the data contain a significant number of false reads from undigested HindIII sites, which can be computationally removed as back-to-back read pairs next to the same restrictive sites (Supplementary Fig. 4c). We also filtered out trans- reads (two ends map to different chromosomes) because a big proportion of trans- reads often reflects high rate of random ligations ${ }^{9,14,19}$. After data filtering, we found that the yield of cis-contacts from eHi-C libraries, especially the ones prepared with in situ ligation procedure, is better than most of the published $\mathrm{Hi}-\mathrm{C}$ libraries prepared with HindIII-based protocol (Fig. 1c, Supplementary Table 1-2). Importantly, the contact 
heatmaps from $\mathrm{Hi}-\mathrm{C}$ and eHi-C data are identical showing the same component $A / B^{1}$ and $\mathrm{TAD}^{3}$ structures (Fig. 1d-e). All these results demonstrated that eHi-C is a reliable alternative to $\mathrm{Hi}-\mathrm{C}$, and can correctly identify 3D genome features from small cell populations.

We also analyzed the intrinsic biases that may affect the eHi-C experimental procedure. Firstly, both $\mathrm{Hi}-\mathrm{C}$ and eHi-C show a decay of contact frequency with increasing distance, but eHi-C captures more short range contacts in $1 \mathrm{Mb}$ range, presumably within TADs (Fig. 2a). Secondly, the contact frequencies involving very short HindIII restriction fragments (<200bp) are lower in both $\mathrm{Hi}-\mathrm{C}$ and eHi-C libraries, which can be explained by the spatial hindrance for those fragments to ligate ${ }^{20}$ (Fig. 2b). Interestingly, although eHi-C appears to preferentially captures contacts between mid-sized fragments (250 1000bp), it has an overall alleviated bias compared to Hi-C, possibly because eHi-C uses more efficient sticky-end ligation instead of blunt-end ligation (Fig. 2b). Thirdly, we also observed GC-bias in eHi-C library, but it is intriguing that the profile of enrichment/depletion is opposite to what was observed for $\mathrm{Hi}-\mathrm{C}^{20}$ (Fig. 2c). We speculate that this might be because both ends of the eHi-C library starting from fixed HindIII restrictive sequence (AGCTT): the GC-bias in eHi-C reflects the efficiency of DNA polymerase elongation after it has already gone through first few bases during PCR amplification or sequencing. Finally, as expected, the length of ligation product will also affect the efficiency of DNA self-circularization (Fig. 2d). These results provide a basis for the eHi-C data normalization and computational inference of DNA contacts. 
To summarize, we have developed a novel easy Hi-C method for convenient mapping of 3D genome architecture. It relies on a highly efficient biotin-free strategy to enrich ligation junctions and therefore is capable of generating high quality DNA library from as few as 0.1 million cells. This low-input eHi-C approach will facilitate the study of genome architecture in rare tissues or small cell populations which are previously unachievable with conventional Hi-C technology.

\section{FIGURE LEGENDS}

\section{Figure 1. Easy Hi-C map DNA contacts genome-wide.}

a, The scheme of easy Hi-C. b, Data filtering results of one exemplary eHi-C library generated from $0.1 \mathrm{M}$ cells. c, Compare the yield of cis-contact reads between 57 published Hi-C libraries and 10 eHi-C libraries. The 4 red spots are eHi-C libraries prepared under in situ ligation condition. d, Heatmaps of contact matrices (chr17) from $\mathrm{Hi}-\mathrm{C}$ and eHi-C at $200 \mathrm{~kb}$ resolution. e, Heatmaps of contact matrices from $\mathrm{Hi}-\mathrm{C}$ and eHi-C at $50 \mathrm{~kb}$ resolution. The top track is drawn using a published IMR90 Hi-C dataset with $\sim 3$ billion reads ${ }^{8}$. A track of TAD structures is plotted in green.

Figure 2. Systematical biases in eHi-C experiments.

a, Curves showing the decay of cis-contact with an increasing distance between two HindIII restrictive fragments. Only "same-strand" reads (see Methods) were used to 
plot the curves. b, Compare the bias from HindIII fragment length in $\mathrm{Hi}-\mathrm{C}$ (left) and eHiC (right) data. All the fragments are binned into 40 equal-sized groups, and the enrichment of trans reads between any two groups are plotted as heatmaps. The enrichment value is defined by the ratio between actual read counts and the global average for any two groups. c, Similarly, HindIII ends are binned into 20 groups based on GC content, and the enrichment of trans- reads are also plotted as heatmaps. For Hi-C (left) we used the GC content in the 200bp region upstream the HindIII site, and for eHi-C, we used the GC content of the region between the HindIII and its nearest Dpnll site. d, Curve shows the average contact frequency from eHi-C against the length of ligation junction products forming DNA circles.

\section{METHODS}

\section{Cell culture and fixation}

Human primary IMR90 fibroblasts were grown as previously described ${ }^{8}$. After confluence, the cells were detached with trypsin and collected by spinning down at $900 \mathrm{~g}$ for 5 minutes. Then the cells were fixed in $1 \%$ formaldehyde for 15 minutes at $37^{\circ} \mathrm{C}$, followed by $1 / 20$ volume of $2.5 \mathrm{M}$ glycine at room temperature for 5 minutes to quench formaldehyde. The fixed cells were scrapped, washed in PBS and pelleted before stored in $-80^{\circ} \mathrm{C}$.

\section{Easy Hi-C}


In this study low-input eHi-C libraries were prepared in two settings. In the first scenario ("aliquot" setting), we started with 1 million IMR90 cells and go through the protocol described below and usually resulted in 250 500ng DNA for library preparation (Fig. 1a). $10 \%$ or $20 \%$ of these DNA were used to generate library $(0.1$ or 0.2 million cells per library). In the second scenario ("mini" setting), we started the experiments with lysing 0.1 or 0.2 million cells following the same protocol as described below, except that all steps before library preparation were performed in $25 \%$ volume. Because the cell lysis and HindIII digestion conditions in this work are different from the published in situ $\mathrm{Hi}-\mathrm{C}$ protocol. We have made modifications in order to ensure nuclei integrity during ligation.

Cell lysis, HindIII digestion, and in situ ligation. Cell pellet from 1 million cells was lysed in $1 \mathrm{ml}$ cell lysis buffer (10mM Tris-Cl, pH7.5, 10mM NaCl, 0.2\% NP-40, 1 X proteinase inhibitor cocktail (Roche)) before incubating on ice for 15 minutes. If there is cell clump in the tube, we dounce the cells for 10 times every cycle for 3 cycles, with one-minute on ice between each cycle. After douncing, the nuclei were put on ice for another 5 minutes and then pelleted by centrifuging $\left(5,000\right.$ rpm for 5 minutes at $\left.4^{\circ} \mathrm{C}\right)$. The pellets were washed once in $1 \mathrm{X}$ Cutsmart buffer (NEB) before resuspended in 360ul $1 \mathrm{X}$ Cutsmart buffer. After resuspension, 40 ul of $1 \%$ SDS were added (final $0.1 \%$ ), and the tubes were incubated at $65^{\circ} \mathrm{C}$ for 10 minutes. To quench the SDS, $44 u$ of $10 \%$ Triton X-100 (final 1\%) was then added to each tube. For chromatin digestion, 400U HindIII (NEB, R3104M 100U/ $/$ l) were added to each tube followed by incubation at $37^{\circ} \mathrm{C}$ for 
4 hours. To ensure efficient digestion, another $400 \mathrm{U}$ of HindIII were added to each tube again for overnight digestion. On day 2 , we digested the nuclei for another 4 hours by adding fresh HindIII enzyme (400U). After digestion, the enzyme was inactivated by adding 40 ul of $10 \%$ SDS (final 1\%) to each tube and incubation at $65^{\circ} \mathrm{C}$ for 20 minutes. The digested products were then transferred to a new $15 \mathrm{ml}$ tube and mixed with $3.06 \mathrm{ml}$ 1.15X ligation buffer $\left(75.9 \mathrm{mM}\right.$ Tris- $\mathrm{HCl}$, ph7.5, 5.75mM DTT, $5.75 \mathrm{mM} \mathrm{MgCl}_{2}$ and 1.15mM ATP). 187 ul $20 \%$ Triton X-100 was added to the mixture and incubated at $37^{\circ} \mathrm{C}$ for 1 hour. For ligation, the products were then mixed with 30ul of T4 DNA ligase (Invitrogen, 15224-025, 1U/ul) and incubated at $16^{\circ} \mathrm{C}$ overnight. After ligation, the tubes were put in room temperature for 30 minutes and the nuclei were pelleted by centrifuging at 5,000rpm for 5 minutes. The supernatant was discarded to remove the free DNA and only the nuclei pellets were kept. The nuclei pellet step is skipped in the "dilute" libraries in Supplementary Table 1. The nuclei pellets were then resuspended in $3.06 \mathrm{ml}$ of $1.15 \mathrm{X}$ ligation buffer and mixed with $40 \mathrm{ul}$ of $10 \%$ SDS and $187 \mathrm{ul}$ of $20 \%$ Triton X-100 for nuclear lysis.

Reverse crosslinking, Dpnll digestion and self-ligation. After nuclear lysis, the mixture were then reverse crosslined at $65^{\circ} \mathrm{C}$ overnight after adding $25 \mathrm{ul}$ of $20 \mathrm{mg} / \mathrm{ml}$ proteinase K. DNA were purified with Phenol: Chloroform: Isoamyl Alcohol (25:24:1,

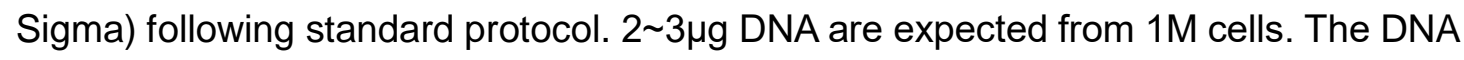

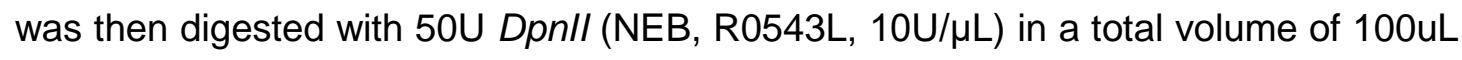
at $37^{\circ} \mathrm{C}$ for 2 hours. After digestion, the enzyme was heat inactivated at $65^{\circ} \mathrm{C}$ for 25 
minutes. The mixture was first incubated with 0.5 volume of PCRClean DX beads (Aline Biosciences) at room temperature for 10 minutes before harvest the supernatant according to vendor's protocol. The supernatant was then incubated with 2 volume of PCRClean DX beads at room temperature for 10 minutes. DNA on the beads were then harvested in $300 u$ un nuclease free water. The two-step bead purification results in DNA with a size range $100 \sim 1,000 \mathrm{bp}$. The DNA products were then mixed with $200 \mathrm{ul}$ of 5X ligation buffer, 5U T4 DNA ligase (Invitrogen) and water to a total volume of $1 \mathrm{ml}$. Self-ligation was done by incubating the tubes at $16^{\circ} \mathrm{C}$ overnight.

Exonuclease digestion and DNA circle re-linearization. The self-ligated DNA were purified again with Phenol: Chloroform: Isoamyl alcohol and digested with $6 \mathrm{U}$ of lambda exonuclease (NEB, M0262S) in $200 \mu \mathrm{L}$ volume at $37^{\circ} \mathrm{C}$ for 30 minutes. The exonuclease was then inactivated by incubating at $65^{\circ} \mathrm{C}$ for 20 minutes. Resulting DNA were purified with 2 volume of PCRClean DX beads as described above. For DNA circle re-linearization, bead bound DNA were eluted and digested with 20U HindIII again at $37^{\circ} \mathrm{C}$ for 2 hours in $150 \mu \mathrm{L}$ volume. The HindIII enzyme was inactivated at $65^{\circ} \mathrm{C}$ for 20 minutes, and the DNA was purified with 2 volume PCRClean DX beads for another time as described above. In the end, bead-bound DNA was eluted in 50ul nuclease free water. From 1M cells, we expect 250-500ng DNA in the end.

Library preparation. We took $10 \sim 20 \%$ of re-linearized DNA ( 50 ng) for library generation following Illumina TruSeq protocol. Briefly, the DNA was firstly end repaired 
using End-it kit (Epicentre). The end-repaired DNA was then A tailed with Klenow fragment ( $3^{\prime}-5^{\prime}$ exo-; NEB) and purified with PCRClean DX beads. Bead bound DNA were eluted in $20 \mu \mathrm{L}$ water and then reduced to $4 \mu \mathrm{L}$ using Speedvac at $50^{\circ} \mathrm{C}$. The $4 \mathrm{ul}$ DNA product was mixed with $5 u$ of $2 X$ quick ligase buffer, $1 \mathrm{ul}$ of 1:10 diluted annealed adapter and 0.5ul of Quick DNA T4 ligase (NEB). The ligation was done by incubating at room temperature for 15 minutes and the enzyme was then inactivated by incubating at $65^{\circ} \mathrm{C}$ for 10 minutes. DNA was then purified with 1.8 volume of DX beads as described above. Elution was done in 14ul nuclease free water. When we check eHiC libraries quality, we only need to sequence less than 1 million reads on MiSeq (Illumina). Because the proportion of PCR duplicates from low-depth sequencing is very low, we used TruSeq indexed adapters (Illumina) without UMI barcode. When we need to deep sequence an eHi-C library, we used custom TruSeq adapter in which the index is replace by 6 base random sequence. The custom adapter was generated by annealing the following two oligos:

Universal oligo -

AATGATACGGCGACCACCGAGATCTACACTCTTTCCCTACACGACGCTCTTCCGA $\mathrm{TC} * \mathrm{~T}$

UMI oligo -/5Phos/GATCGGAAGAGCACACGTCTGAACTCCAGTCACNNNNNNATCTCGTATG CCGTCTTCTGCTT* ${ }^{\star}$

PCR amplification of DNA libraries. To amplify the DNA libraries, we mixed 13ul adapter 
ligated DNA with 1ul of 20uM oligo C (AATGATACGGCGACCACCGAGATCTACAC), 1ul of 20uM oligo D (CAAGCAGAAGACGGCATACGAGAT) and 15ul of 2X KAPA HiFi Hotstart ready mix (Kapa Biosystems). And the PCR amplification was done as follows: denature at $98^{\circ} \mathrm{C}$ for 45 seconds, cycled at $98^{\circ} \mathrm{C}$ for 15 seconds, $60^{\circ} \mathrm{C}$ for 30 seconds, $72^{\circ} \mathrm{C}$ for 30 seconds, and did 5 cycles at first for estimating the total cycle number needed, and then further extension at $72^{\circ} \mathrm{C}$ for 5 minutes. The products were then purified using 1.8 volume of DX beads to remove primer contamination as described above. And the DNA was eluted in 20ul nuclease free water. And library quantification was done following the protocol of Illumina library quantification kit (KAPA Biosystems, KK4824). PCR was done again in $50 \mu \mathrm{L}$ volume for a target final concentration 20 40nM (usually 3 4 additional cycles). The generated libraries were then subjected to sequencing.

\section{Analysis of Hi-C and eHi-C data}

Alignment and removing PCR duplications. Published IMR90 Hi-C data were used in this study to compare with eHi-C. The accession numbers of $\mathrm{Hi}-\mathrm{C}$ data are listed in Supplementary Table 2. All the sequencing data were mapped to human reference genome hg18 using Bowtie. For Hi-C, the two ends of paired-end (PE) reads were mapped independently using the first 36 bases of each read. PCR duplications were defined as PE reads with both ends mapped to the same locations. For eHi-C, because nearly all the mappable reads start with HindIII sequence AGCTT, we trimmed the first 5 bases from every read, took the next 36 bases, and add the 6-base sequence 
AAGCTT to the 5' of each reads before mapping using the whole 42 bases. Some MiSeq runs were performed with reads shorter than 41 bases, and the full length reads will be used in those cases. After mapping, we further filter the reads requiring the positions of both ends to be exactly at the HindIII cutting sites. Because the eHi-C library deep sequenced was prepared with UMI adapter, PCR duplications were defined as identical PE reads also with the same UMI barcode. The eHi-C libraries sequenced on MiSeq were not intended for deep sequencing and therefore do not have UMI barcode. PCR duplications in those libraries were removed the same way as $\mathrm{Hi}-\mathrm{C}$.

QC analysis of Hi-C and eHi-C libraries. After remove PCR duplications, we analyzed the library quality by classifying the reads into different categories. In both $\mathrm{Hi}-\mathrm{C}$ and eHi-C, the percentage of trans- contacts can be easily calculated by count the number of reads with two ends on different chromosomes (listed in Supplementary Table 12). For cis- reads in $\mathrm{Hi}-\mathrm{C}$ data, we first discard the reads with both ends mapped to the same HindIIl fragments as invalid pairs. Dangling ends are defined as "inward" pairs among the invalid pairs (Supplementary Fig. 4a) and the percentage are listed in Supplementary Table 2. The rest invalid pairs are classified into "other false" category. Since cut-and-ligation events are expected to generate reads within 500bp upstream of HindIII cutting sites due to the size selection ("+" strand reads should be within 500bp upstream of a HindIII site, and "-" strand reads should be within 500bp downstream a HindIII site), we only keep reads pairs with both ends satisfying this criteria. The other 
pairs are also classified into "other false" category in Supplementary Table 2. After this step, every remaining paired-end reads represents one pair of restrictive fragments. We next split all these reads into three classes based on their strand orientations ("same-strand", "inward", or "outward"). We have previously shown that although theoretically "same-strand" reads should be twice as many as "inward" or "outward" reads, in reality more "inward" or "outward" reads can be observed due to incomplete digestion of chromatin ${ }^{8}$. We therefore estimate the total number of real cis-contact as twice the number of valid "same-strand" pairs, the percentages are listed in Supplementary Table 2. For eHi-C, library, the only type of invalid cis- pairs are selfcircles with two ends within the same HindIII fragment facing each other (Supplementary Fig. 4b). Similar to Hi-C, we also computed the total number of real cis-contact as twice the number of valid "same-strand" pairs. Reads from undigested HindIII sites are back-to-back read pairs next to the same HindIII sites facing away from each other (Supplementary Fig. 4c).

Bias analysis of Hi-C and eHi-C libraries. To plot the decay of contact with distance (Fig. 2a), we only used "same-strand" cis-contact reads. For any given distance $L$, we found all HindIII fragment pairs with gap distance between $0.9 * L$ and $1.1 * L$, and computed the average contact frequency among them. We normalize these numbers by dividing them by the average contacts from all the intra-chromosome HindIII fragment pairs. We used trans- contact data to compute the fragment length and GCcontent bias because in this way, the distance is no longer a parameter of concern. 
For length bias (Fig. 2b), we divided all the HindIII fragments into 40 equal-sized groups and computed the average contact frequency for each pair of groups, and enrichment values were calculated by normalizing to global average. Similarly, we plot the GC bias (Fig. 2c) by dividing all the HindIII ends into 20 equal-sized groups by GC content. For Hi-C, the GC content was computed using the 200bp near each HindIII end. For eHi-C, the GC content was computed for the region between a HindIII end and the nearest Dpn/l site.

Data availability. Raw and processed eHi-C data are available at NCBI GEO with accession number GSExxxxx.

\section{REFERENCES}

1. Lieberman-Aiden, E. et al. Comprehensive mapping of long-range interactions reveals folding principles of the human genome. Science 326, 289-293 (2009).

2. Denker, A. \& de Laat, W. The second decade of 3C technologies: detailed insights into nuclear organization. Genes Dev 30, 1357-1382 (2016).

3. Dixon, J.R. et al. Topological domains in mammalian genomes identified by analysis of chromatin interactions. Nature 485, 376-380 (2012).

4. Nora, E.P. et al. Spatial partitioning of the regulatory landscape of the X-inactivation centre. Nature 485, 381-385 (2012).

5. Pope, B.D. et al. Topologically associating domains are stable units of replication-timing regulation. Nature 515, 402-405 (2014).

6. Dixon, J.R. et al. Chromatin architecture reorganization during stem cell differentiation. Nature 518, 331-336 (2015).

7. Naumova, N. et al. Organization of the mitotic chromosome. Science 342, 948-953 (2013).

8. Jin, F. et al. A high-resolution map of the three-dimensional chromatin interactome in human cells. Nature 503, 290-294 (2013).

9. Rao, S.S. et al. A 3D map of the human genome at kilobase resolution reveals principles of chromatin looping. Cell 159, 1665-1680 (2014).

10. Beagan, J.A. et al. Local Genome Topology Can Exhibit an Incompletely Rewired 3D-Folding State during Somatic Cell Reprogramming. Cell Stem Cell 18, 611-624 (2016).

11. de Wit, E. et al. The pluripotent genome in three dimensions is shaped around pluripotency 
factors. Nature 501, 227-231 (2013).

12. Kagey, M.H. et al. Mediator and cohesin connect gene expression and chromatin architecture. Nature 467, 430-435 (2010).

13. Phillips-Cremins, J.E. et al. Architectural protein subclasses shape 3D organization of genomes during lineage commitment. Cell 153, 1281-1295 (2013).

14. Belton, J.M. et al. Hi-C: a comprehensive technique to capture the conformation of genomes. Methods 58, 268-276 (2012).

15. Simonis, M. et al. Nuclear organization of active and inactive chromatin domains uncovered by chromosome conformation capture-on-chip (4C). Nat Genet 38, 1348-1354 (2006).

16. Nagano, T. et al. Single-cell Hi-C reveals cell-to-cell variability in chromosome structure. Nature 502, 59-64 (2013).

17. Nagano, T. et al. Comparison of Hi-C results using in-solution versus in-nucleus ligation. Genome Biol 16, 175 (2015).

18. Tanizawa, H. et al. Mapping of long-range associations throughout the fission yeast genome reveals global genome organization linked to transcriptional regulation. Nucleic acids research 38, 8164-8177 (2010).

19. Kalhor, R., Tjong, H., Jayathilaka, N., Alber, F. \& Chen, L. Genome architectures revealed by tethered chromosome conformation capture and population-based modeling. Nature biotechnology 30, 90-98 (2012).

20. Yaffe, E. \& Tanay, A. Probabilistic modeling of Hi-C contact maps eliminates systematic biases to characterize global chromosomal architecture. Nat Genet 43, 1059-1065 (2011). 


\section{Figure 1}

a

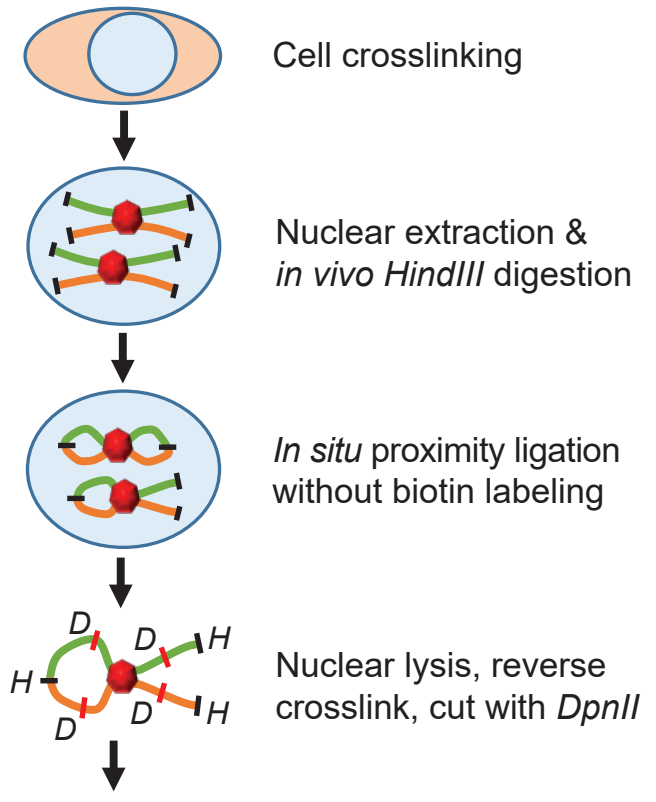

Expected DNA species:
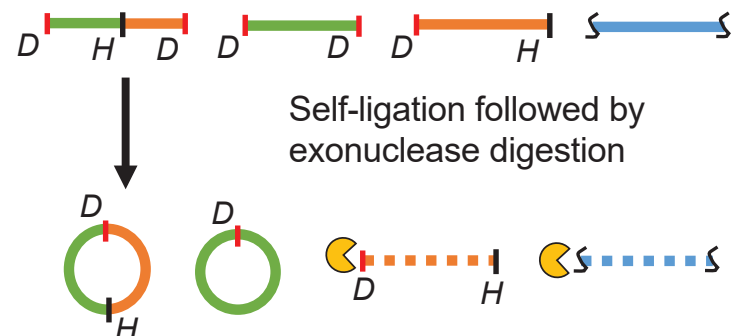

1 Re-linearize junction products with $2^{\text {nd }}$ round HindIII digestion

$H^{\prime} D^{\prime} H^{\prime}$

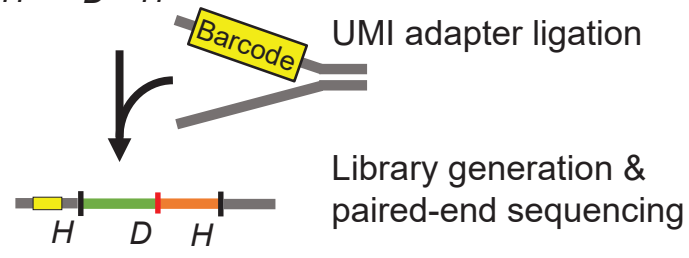

b
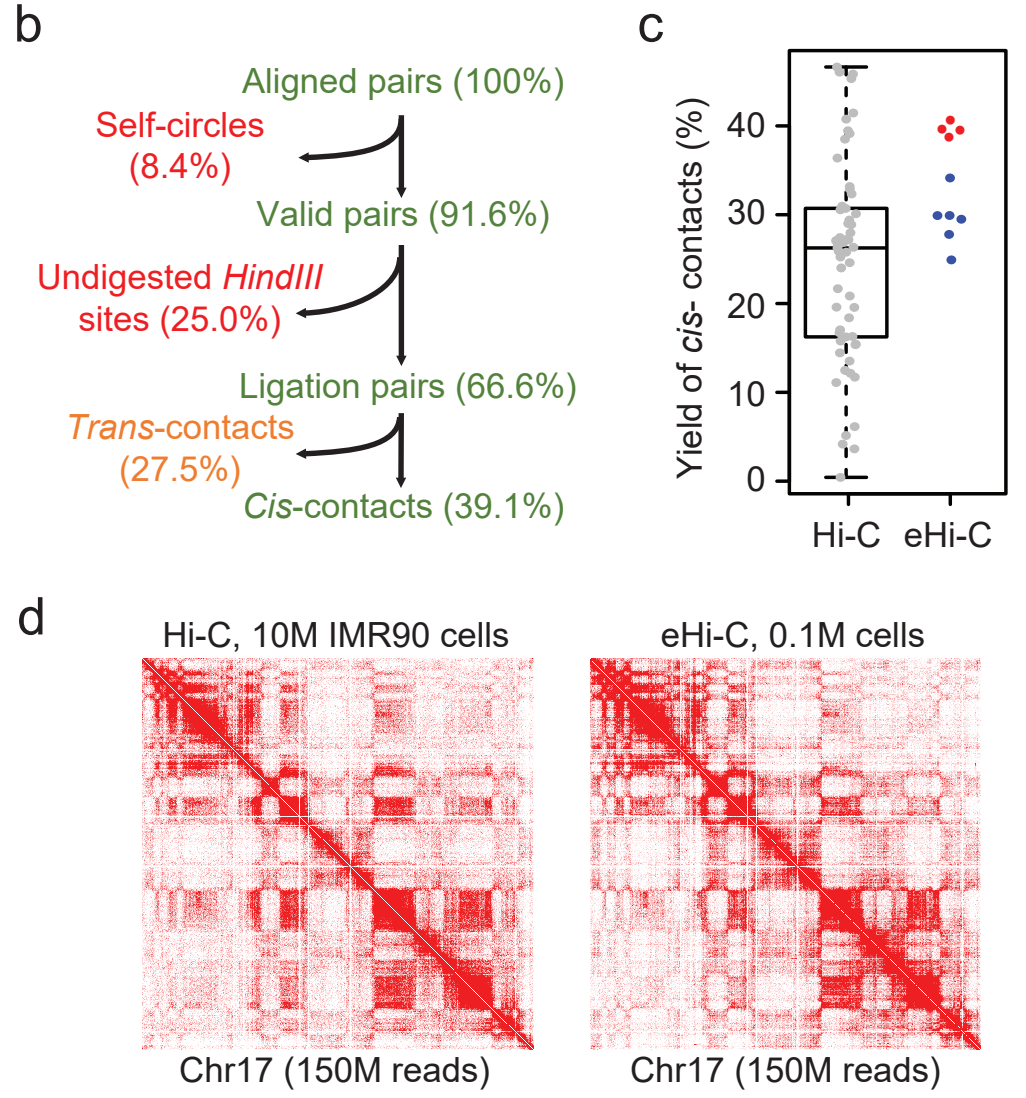

e

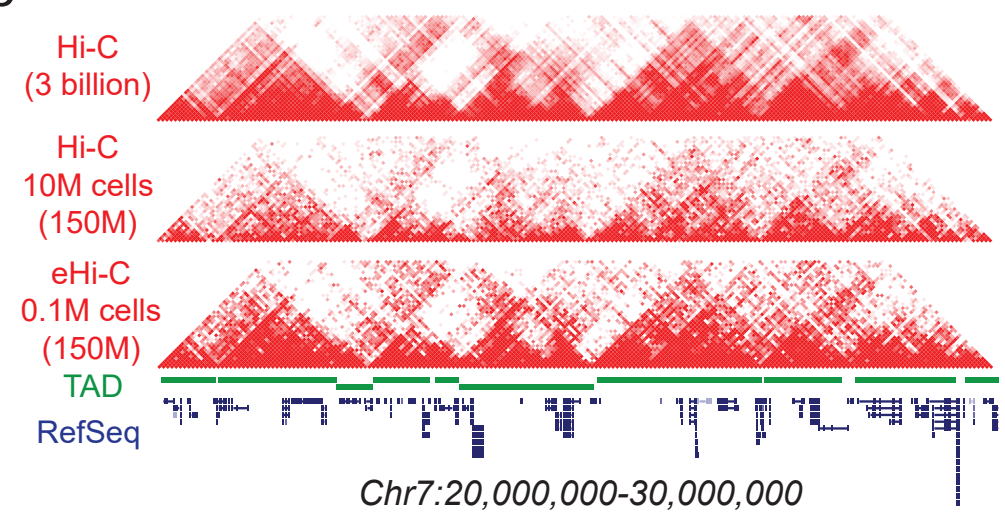




\section{Figure 2}

a

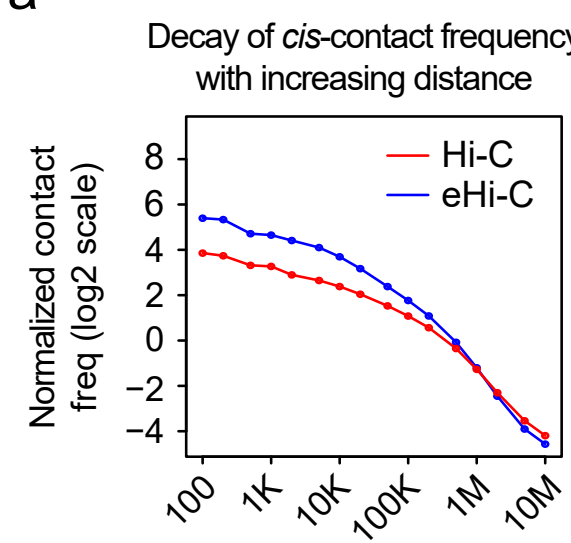

Distance (bp)

C

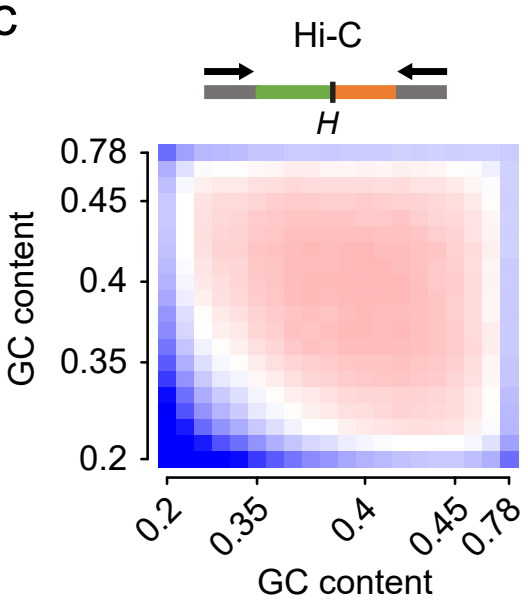

b

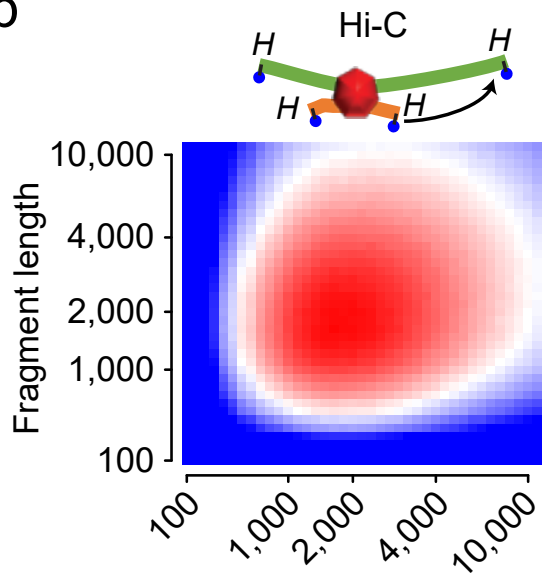

Fragment length

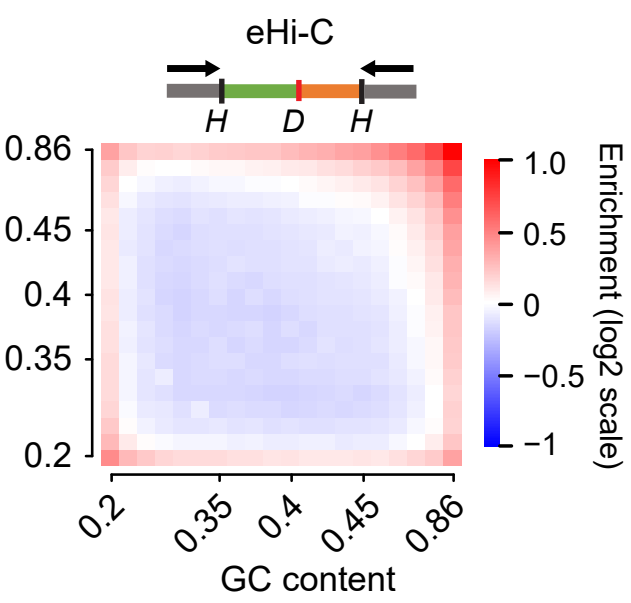

d

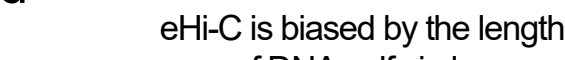
of DNA self circles

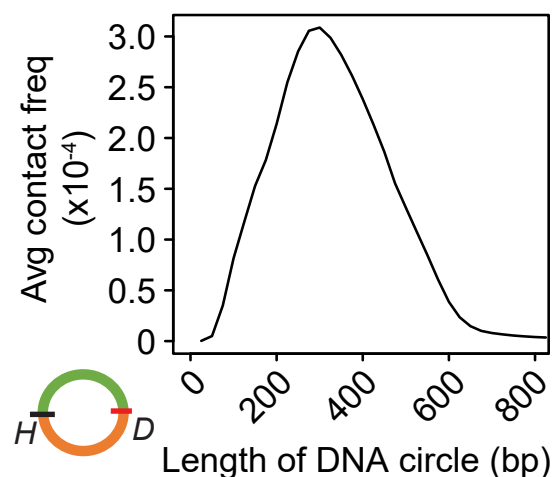

Length of DNA circle (bp)

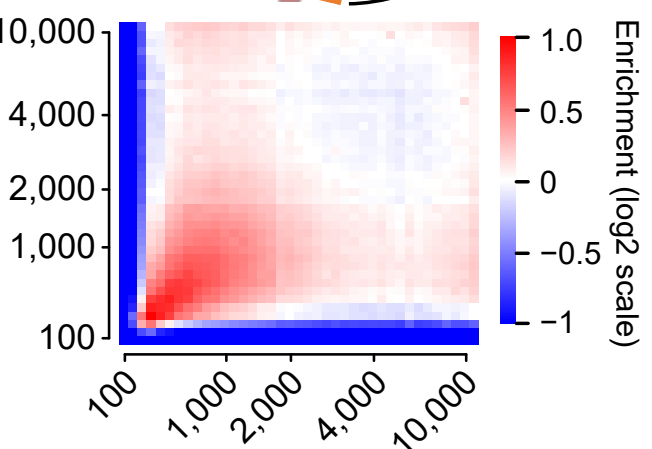

Fragment length 